\title{
Estimation of Forces Acting on a Sailboat Using a Kinematic Sensor
}

\author{
Alan Mahne Kalin, Dejan Žagar, Peter Vidmar
}

Amateur keel boat racing has become increasingly popular in coastal regions with a long sailing tradition, such as the Adriatic. Although the traditional experience is transferred to new generations of skippers and crews that compete in national and international regattas with open class boats, the material limits of the boat are often measured subjectively, by sail or mast failure, and transferred through a story. Most sailors know why a component failed, but often they do not know what force was needed for the particular failure. Forces acting on a boat are usually estimated with CFD and towing tank experiment for relevant sailing conditions, but full scale data in seaway are rarely taken. Here we want to show a low budget method to get a rough estimate of aerodynamic and hydrodynamic forces acting on a keel sailboat using a kinematic sensor. Some approximations are taken into account to construct a simplified mathematical sailboat model, which allow to relate kinematic data to forces acting on the sail, hull, keel, and rudder. Some data such as the

\section{KEY WORDS \\ $\sim$ Sailing boat \\ $\sim$ Inverse dynamic \\ $\sim$ Broach \\ $\sim$ Forces \\ $\sim$ Kinematic sensor}

\footnotetext{
University of Ljubljana, Faculty of maritime studies and transport

e-mail: alan.mahne.kalin@fpp.uni-lj.si

doi: 10.7225/toms.v07.n02.002

This work is licensed under (cc) BY
}

geometry and mass distribution of the boat has to be known; other parameters such as water resistance instead has to be experimentally measured. The results of a series of measurements are presented and discussed. Looking on the limitations of such a method, a proposal for a new sensor is made.

\section{INTRODUCTION}

An inverse dynamic approach was attempted to evaluate the forces and moments acting on a sailboat while underway. The sailboat is a $10 \mathrm{~m}$ inshore racer Evolution 10 designed by Andrej Justin. Forces and torques arising from the weather effects on hull, keel, rudder, and sails were modelled based on experimental measuring and empirical equations. The input data to the model has been later taken measuring kinematic and rudder position.

The obtained data depends heavily on the weather conditions and crew reactions. Also, in controlled conditions repeated experiments will give different measurements. In such an approach, there are many kinds of errors to take into account when working out the solution.

For simplicity's sake, an indexing system will be used to depict the forces, torques and arms: the first index reveals the nature, the second reveals the axis.

Two coordinate systems are used: inertial and body-fixed. The inertial system is fixed: the origin coincides with the first measured position on the water level, axis 4 is toward north, 5 is toward west, and 6 completes the right-hand system toward zenith (up). The body coordinate system is fixed to the sailboat: the origin coincides with the boat's center of gravity (CG), axis 1 is toward bow parallel to the design water plane, axis 2 is toward port side parallel to the design water plane, and axis 3 completes the right-hand system. 
Table 1.

Indexing system.

\begin{tabular}{llll} 
First index & Nature & $\begin{array}{l}\text { Second } \\
\text { index }\end{array}$ & axis \\
\hline 1 & $\begin{array}{l}\text { Wind effect } \\
\text { on sails }\end{array}$ & 1 & Body-x \\
\hline 2 & $\begin{array}{l}\text { Water effect } \\
\text { on hull }\end{array}$ & 2 & Body-y \\
\hline 3 & $\begin{array}{l}\text { Water effect } \\
\text { on keel }\end{array}$ & 3 & Body-z \\
\hline 4 & $\begin{array}{l}\text { Water effect } \\
\text { on rudder }\end{array}$ & 4 & Inertial-North \\
\hline 5 & $\begin{array}{l}\text { Sea waves } \\
\text { effect on hull }\end{array}$ & 5 & Inertial-West \\
\hline & & 6 & Inertial-Up \\
\hline
\end{tabular}

Two coordinate systems are used: inertial and body-fixed. The inertial system is fixed: the origin coincides with the first measured position on the water level, axis 4 is toward north, 5 is toward west, and 6 completes the right-hand system toward zenith (up). The body coordinate system is fixed to the sailboat: the origin coincides with the boat's center of gravity (CG), axis 1 is toward bow parallel to the design water plane, axis 2 is toward port side parallel to the design water plane, and axis 3 completes the right-hand system.

\subsection{Equations of Motion}

Equations of motion expressed for the body coordinate system are:

$\sum_{i=1}^{5} F_{i, j}=m a_{j} \quad j=1,3$

$\sum_{i=1}^{5} T_{i, j}=I_{j, k} a_{k}+\Omega_{j, k} I_{k, m} \omega_{m} \quad j=1,3$

where $F_{i, j}, m, a_{j}, \tau_{i, j} I_{k, m}, a_{j}, \omega_{j}$ and $\Omega_{j, k}$ are external forces due to $i$ acting along $j$, mass, linear acceleration, external torque due to $i$ acting along $j$, mass inertia matrix, angular acceleration, angular velocity and angular velocity skew symmetric operator respectively.
The linear and angular acceleration and velocities were measured by the motion tracking device (MT) that was fixed off the body CG. Such offset gives rise to a centrifugal term due to angular velocity and a tangential acceleration term due to angular acceleration. The maximum calculated value for centrifugal term and tangential acceleration term was $0.02 \mathrm{~m} / \mathrm{s} \wedge 2$, and $0.2 \mathrm{~m} / \mathrm{s} \wedge 2$ respectively, where the sensor noise level was $0.15 \mathrm{~m} / \mathrm{s} \wedge 2$. For this reason, these two terms were neglected.

\subsection{Modelling the Inertia Matrix}

The geometry of the boat was measured while docking, picking various point coordinates on the hull and the appendages. The points where then plotted in CAD software and a surface was fitted to them. The deck and main interior structures were also designed, and then an area inertia matrix was calculated from the software for each component (surface). A surface density was assigned to each component to get a mass inertia matrix.

The exact surface density of the boat was not known for each part, so an experimental measurement was done to estimate the $I_{1,1}$ and then surface density was manually assigned to the CAD model to fit the measured values of $I_{1,1}$ while keeping the CG of the assembly at the origin. The inertia matrix was then calculated for all the other terms using the surface density distribution.

The experiment to estimate $I_{1,1}$ was performed as a damped oscillation around axis 1 . The following analytical solution was fitted to the results obtained from MT

$\Phi_{1}(t)=A e^{(-\beta t)} \cos \left(\omega_{1} t-\varphi\right)+d$

to get the amplitude $A$, damping $\beta$, damped angular frequency $\omega_{1}$, phase offset $\varphi$ and roll offset $d$ of roll respect to time $\Phi_{1}(t)$. If neutral axis of the body are aligned with body coordinate system, then $I_{1,1}$ is related to the previous parameters through

$I_{1,1}=\frac{k m g}{\omega^{2}{ }_{1}+\beta^{2}}$

where $k$ is the first derivative of righting arm with respect to the roll angle in the linear region near zero. The results of the fit are shown in the following Figure 1. 


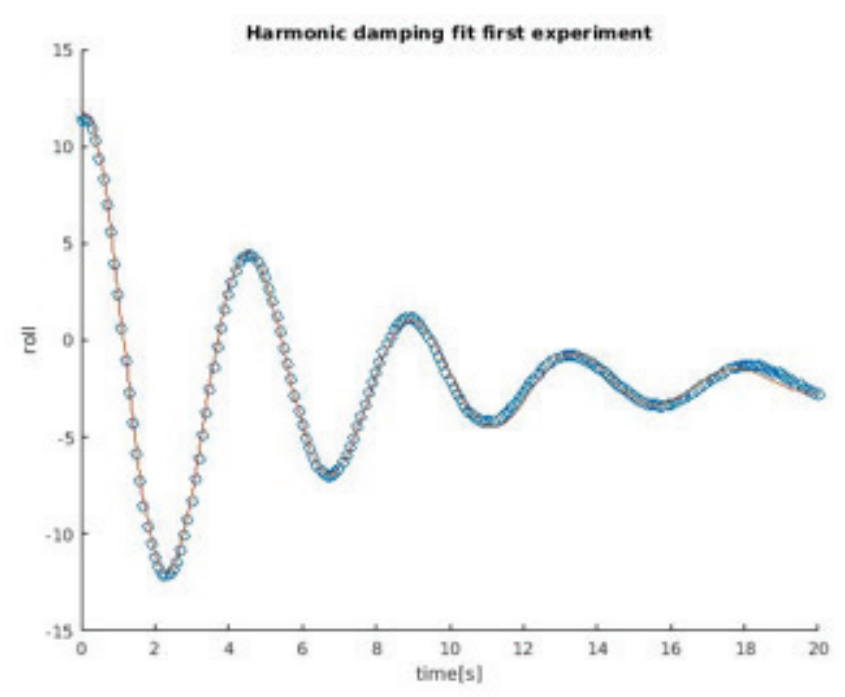

Figure 1.

Experimental measurements of harmonic damping $(12 / 01 / 2018)$ - roll angle expressed in degrees

\subsection{Hull Water Resistance Model}

Hull resistance was measured by towing and stopping experiments. The towing experiment was performed using a dynamometer placed between the boat's mast and the towing line, towing the boat at speeds up to $15 \mathrm{kts}$. The stopping experiment was performed towing the boat to approximately $7 \mathrm{kts}$ and measuring the velocity profile while stopping after the towing line release. All the measurements were taken in calm seas with no real wind, for several headings.

Boat's resistance is assumed to be composed of parasitic drag (form drag, skin friction and interference drag), wave drag, and lift induced drag. Parasitic drag was modeled using the square law

$$
F_{R 1}\left(v_{1}\right)=-a\left(v_{1}\right) v_{1}^{2}
$$

the wave drag was modeled using the analytical solution from (Fitzpatrick, 2018).

$$
F_{R 2}\left(v_{1}\right)=-b\left[\frac{\sin \left(\pi-F r^{-2}\right)}{\pi-F r^{-2}} \frac{1}{1+\pi F r^{-2}}\right]^{2}
$$

$$
\text { where } F r \text { is the Froude number } \quad F_{r}=\frac{v 1}{\sqrt{g l}}
$$

$v_{1}$ is the linear velocity along the axis $1, g$ is gravitational acceleration, and $I$ is the length of the waterline.

Together the analytic equation fitted to measured data is (Mahne Kalin, 2014)

$$
F_{2,1}\left(v_{1}\right)=-a\left(v_{1}\right) v_{1}^{2}-b\left[\frac{\sin \left(\pi-F r^{-2}\right)}{\pi-F r^{-2}} \frac{1}{1+\pi F r^{-2}}\right]^{2}
$$

where

$$
\begin{aligned}
a\left(v_{1}\right)=43.3 & -2.705\left(\frac{\pi}{2}+\arctan \left(\frac{v_{1}-4.62}{3.79}\right)\right) \\
& +13.05\left(\frac{\pi}{2}+\arctan \left(\frac{v_{1}-3.94}{0.75}\right)\right)
\end{aligned}
$$

and

$$
b=3990
$$

\subsection{Keel and Rudder Models}

To check the feasibility, two approaches were taken to model keel and rudder.

The first approach uses lift and drag coefficients based on potential fluid dynamic program that are freely available on the internet. Those have been computed for $2 \mathrm{D}$ profiles and a correction is needed to account for the induced drag due to the finite length of the profile.

The second approach uses an empirical formula shown in (Larsson, Eliasson and Orych, 2014). It was developed based on data from several tank tests.

The program uses the first approach to model the keel and rudder, while the second approach is used for comparison.

\section{The first method}

Lift and drag coefficients were taken from the data table available on (AirFoil Tools, 2018). The profiles in question are NACA 0012 for the rudder and NACA 63015A for the keel. The coefficients are calculated for 2D geometry, so 3D effects, such as the induced drag, have to be accounted for.

The coefficients are tabulated in the program, and an interpolation function is used to get the desired value of the angle of attack (AOA). 
The corrections used to calculate the 3D lift are:

$C_{L 3 D}=C_{L 2 D}\left(\frac{A R}{A R+2}\right)$

$C_{D 3 D}=C_{D 2 D}\left(\frac{C^{2}}{\pi A 2 D}\right)$

where $C_{L 2 D}$ and $C_{D 2 D}$ are the two-dimensional lift and drag coefficients respectively, and $A R$ is the aspect ratio.

Finally, the forces due to the rudder and keel are:

$F_{3,2}=0.5 \rho V^{2} A_{k}\left(\cos (\beta) C_{k, L 3 D}+\sin (\beta) C_{k, D 3 D}\right)$

$F_{4,2}=0.5 \rho V^{2} A_{r}\left(\cos (\beta) C_{r, L 3 D}+\sin (\beta) C_{r, D 3 D}\right)$

\section{The second method}

The derivative of the lift coefficient for small angles is defined by:

$\frac{d C_{L}}{d_{a}}=\frac{5.7 \cdot A R_{e}}{1.8+\cos (\lambda) \cdot \sqrt{\left(\frac{A R_{e}^{2}}{\cos ^{4}(\lambda)}+4\right)}}$

$A R_{e}=2 \cdot A R$

$a_{k}=\beta$

$a_{r}=\beta-\varepsilon+\delta_{r}$

$\varepsilon=0.136 \cdot\left(\frac{C_{L, K}}{A R_{e, k}}\right)^{0,5}$ where $A R$ is the aspect ratio of the fin, $\lambda$ is the sweep angle; $\beta, a_{k^{\prime}}$ $a_{r}$ and $\delta_{r}$ are leeway angles, AOA of the keel, AOA of the rudder and rudder angle respectively, all expressed in radians. The index $k$ and $r$ stand for the keel and rudder respectively. The integral of the equation (14) is

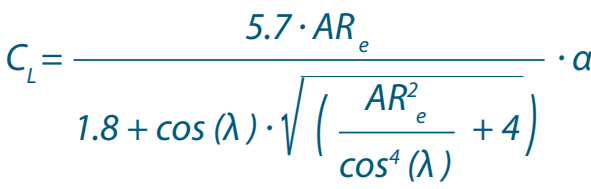

The lift coefficients are multiplied by the dynamic pressure and the respective planform area.

$L_{k}=C_{L, k} \cdot 0.5 \cdot \rho \cdot V^{2} \cdot A_{k}$

$L_{r}=C_{L, r} \cdot 0.5 \cdot \rho \cdot(0.9 \cdot V)^{2} \cdot A_{r}$

Note that for the rudder case only $90 \%$ of the water velocity is accounted due to the keel interference.

The hydrodynamic side force:

$F_{3,2}=L_{k} \cdot C_{\text {hull }} \cdot C_{\text {heel }}$

$F_{4,2}=L_{r} \cdot C_{\text {hull }} \cdot C_{\text {heel }}$

where $\Phi$ is the roll angle in radians, $T_{c}$ is the draft of the canoe, and $T_{k}$ is the draft of the keel. 
The angle of attack for the keel is the leeway angle, while for the rudder the change in the flow direction due to the keel is considered as well as the rudder angle.

Here, the hydrodynamic side force was calculated directly from the lift, using small angle approximation.

\subsection{Hydrostatic Model}

For the present purpose, the hydrostatic is the main source of torque. Hydrostatic torque acts around the axes 1 and 2 .

Torque around the axis 1 can be estimated knowing the roll angle and crew weight distribution. During the measurements, only 3 crew members were on board and were assigned a fixed position from which a correction was calculated to get the true righting arm. The models used in the program to get the righting moment are based on the geometry and mass distribution for a discrete set of rolling positions. As required by the program, the value of righting arm is interpolated from previously calculated data.

Torque around the axis 2 is very difficult to estimate, as a small change in pitch requires a relatively big amount of torque, and the changes in pitch are comparable with the MT error in estimating the pitch. Therefore, the analysis driven by this data would be chaotic and has been replaced by a holonomic constraint

$\Phi_{2}(t)=0$

The torque necessary to fulfill the equation of motion was assigned to this constraint, appearing as $\tau_{2,2}$. This may seem straightforward, but transforming the torque vector from the boat to the inertial coordinate system $\tau_{2,2}$ acts also as a weather helm when the boat is heeled. This can be a major cause that can provoke a broach.

Hydrostatic forces in calm seas are oriented positively along the axis 6 , while weight force due to gravity is always oriented downwards. Assuming calm seas, the two forces are always equal and opposite, so they are discarded from the analysis.

\section{MEASUREMENTS}

All the forces and torques have to fulfill the equations of motions at all times. The "known" forces and torques arise from the models that are incomplete or at least not always correct. There are factors that can be missing in the modelling process, so there must be space for error.

The data given by the MT are also subject to error that can be intrinsic in the device or due to internal estimate errors. The MT used to get the present data is Xsens MTI/g. It measures directly
3D accelerations, angular velocities, magnetic field orientation and position from the GPS. Raw data are then filtered through a special extended Kalman filter to get the orientation and velocity data in real time. Velocity and orientation are estimated values and it has been noted that they can be delayed with respect to raw data'. Due to slow changes in the dynamic, a $10 \mathrm{~Hz}$ sampling filtered was chosen.

Based on these observations, we opted to derive the angular velocity and acceleration from the filtered position and linear acceleration from the filtered velocity.

\subsection{Time Derivatives of Rotation Matrix and Velocity}

Rotation matrix is formed from Tait-Bryant angles, given as output data from the MT, using the following equation:

$R=R_{3} R_{2} R_{1}$

where

$R_{1}=\left[\begin{array}{ccr}1 & 0 & 0 \\ 0 & \cos \left(\Phi_{1}\right) & -\sin \left(\Phi_{1}\right) \\ 0 & \sin \left(\Phi_{1}\right) & \cos \left(\Phi_{1}\right)\end{array}\right]$

$R_{2}=\left[\begin{array}{llr}\cos \left(\Phi_{2}\right) & 0 & \sin \left(\Phi_{2}\right) \\ 0 & 1 & 0 \\ -\sin \left(\Phi_{2}\right) & 0 & \cos \left(\Phi_{2}\right)\end{array}\right]$

$R_{3}=\left[\begin{array}{ccc}\cos \left(\Phi_{3}\right) & -\sin \left(\Phi_{3}\right) & 0 \\ \sin \left(\Phi_{3}\right) & \cos \left(\Phi_{3}\right) & 0 \\ 0 & 0 & 1\end{array}\right]$

and $\Phi_{i}$ is the rotation angle around the axis $i$. The rotation matrix $R$ is an operator that rotates a vector expressed in inertial frame to body frame. The time derivative of rotation matrix is

$\dot{R}=R \Omega$

1. It has been observed during damped oscillation that tangential acceleration is delayed from orientation. This delay can give rise to errors if we use velocity derivative and acceleration interchangeably. 
from which the components of the skew symmetric operator $\Omega$ can be taken. These are the components of angular velocity vector expressed in body frame $(\omega)$. Numerical derivative has been taken with central difference scheme.

Having the angular velocity vector, the angular acceleration vector has been taken using the central difference scheme.

Before each derivation, a moving average filter with window of 0.5 seconds was applied to the data to avoid the spreading of errors due to noise.

The acceleration was calculated taking the numerical derivative with central difference scheme to velocity, which was filtered using a moving average filter with window of 0.5 seconds.

\subsection{Summary of Forces and Torques}

At this point the kinematic data of the boat are known; some forces have been modeled using kinematic data, and some are unknown. The following equation represents the known or modeled terms on the right hand side and the unknown on the left hand side.

$F_{1, j}+F_{5, j}=-\sum_{i=2}^{4} F_{i, j}+m a_{j} \quad j=1,3$

$\tau_{1, j}+\tau_{5, j}=-\sum_{i=2}^{4} \tau_{i, j}+l_{j, k} a_{k} \quad j=1,3$

As it is, this system has 6 equations and 12 unknowns, so some assumptions have to be made to solve the problem. The last term of the right hand side describes the changing of momentum, and it will be bigger for sudden changes.

The two unknown forces and torques are due to the wind and waves. These two phenomena can be located by the frequency of their changes. Wind changes with a very low frequency with respect to waves. This means that by grouping the two phenomena together the high frequencies can be attributed to waves, and the low frequency can be attributed to sail.

Taking the difference to the limit, we assumed that the sail forces are nearly constant or, in other words, that the measured kinematic term arising due to a change of sail forces only is usually so low that its detection using the MT is comparable to noise level. Assuming this, all the dynamic part can be attributed to the waves and we can rewrite the equations in

$F_{1, j}=-\sum_{i=2}^{4} F_{i, j} \quad j=1,3$

$$
\begin{aligned}
& F_{5, j}=m a_{j} \quad j=1,3 \\
& \tau_{1, j}=-\sum_{i=2}^{4} \tau_{i, j} \quad j=1,3 \\
& \tau_{5, j}=l_{j, k} a_{k} \quad j=1,3
\end{aligned}
$$

The equations (32) and (34) can be solved directly; the equation (31) can also be solved directly, but the accuracy of estimating $F_{1, j}$ is very important as it appears also in the related torque. The equation (34) in its component form runs as follows:

$-d_{1,3} F_{1,2}=d_{3,3} F_{3,2}+d_{4,3} F_{4,2}-\tau_{2,1}$

$\tau_{2,2}+d_{1,3} F_{1,1}=-d_{3,3} F_{3,1}-d_{4,3} F_{4,1}$

$\tau_{2,2} \tan \left(\Phi_{1}\right)+d_{1,1} F_{1,2}=-d_{3,1} F_{3,2}-d_{4,1} F_{4,2}$

Here, the assumption has been taken that all the arms producing the required torques lies on the $x-z$ plane, and that sail, rudder, and keel forces' vectors lie in the $x-y$ plane only. This assumption is reasonable mainly when hoisting jib, genoa or main sail, which are flat and produce no vertical lift. This will not be the case for spinnaker like sails, whose vertical lift is known to help rising the bow from the water.

The equations (35) to (37) have been arranged so that all the unknown terms lie on the left hand side.

If we use the results from the equation (31) in the equations (35), (36) and (37), the only unknowns are $\tau_{2,2}, d_{1,1}$, and $d_{1,3}$.

$\tau_{2,2}$ appears in both equations because the holonomic constraint that prevents changing in pitch acts horizontally in the inertial system, which has to be properly transformed when expressing in body frame. To be clearer, the same torque vector in the inertial frame has components

$q=R_{3}\left[\begin{array}{c}0 \\ \sqrt{\tau_{2,2}{ }^{2}+\left(\tau_{2,2} \tan \left(\Phi_{1}\right)\right)^{2}} \\ 0\end{array}\right]$ 


\subsection{Two Ways to Solve the Same System}

Bearing in mind that the modeled forces are subject to error deriving from non-universality of the model, the system can be solved in two ways (later referred to as methods A and B):

- $\quad$ Assuming the location of the center of effort of sail $d_{1,1}$ and $d_{1,3}$, and then solving for $F_{1,1}$ and $F_{1,2}$. (A)

- Calculating $F_{1,1}$ and $F_{1,2}$, and then solving for $d_{1,1}$ and $d_{1,3} \cdot(B)$

Both methods give a solution and they can be compared; however, they have been found to be very different in some particular cases.

For the rule-of-thumb, the center of effort of sails lies approximately one quarter forward of the geometric center of the area of both sails. At the same time, it has to lie also on the sail projected area. These two conditions can be checked continuously creating a proper fuzzy set.

Direct calculation of $F_{1,1}$ and $F_{1,2}$ (method $B$ ) can lead to errors because of the difficulty in estimating the angle of attack (AOA) acting on the keel and the rudder, as the decisive factor in the keel and rudder force model.

\subsection{Estimation of AOA}

The MT measures the magnetic heading and the velocity over ground. These two measurements are derived independently as the MT does not assume any constraint. The AOA of keel can be estimated only by taking the horizontal angle between unit vectors of velocity and heading. Such method is prone to errors arising from the drift and magnetic variations.

The AOA of rudder is simply assumed to be AOA of keel plus the rudder angle, taking positive when the boat tends to turn right at forward speed.

Note that method A neglects the measured AOA of the keel and implies a calculated one. The calculation of the implied AOA of the keel also requires to take into account the rudder angle. As the solution is not analytically solvable, a Newton-Rhapson method was used to find the proper value of AOA of the keel.

To estimate the accuracy of such a measurement, we computed the required angle of attack to solve for method $A$. The explanation and comments of the results of such a comparison are expressed in the next section.

\section{RESULTS}

A comparison between the required and actual AOA of keel was made using methods $A$ and $B$. They do not agree, and there are more explanations to this.

The required $A O A$ based on this procedure is too low with respect to the one expected: the boat was sailing windward at an angle to the real wind of approximately 45 degrees in fresh air. During the measurements, the time averaged roll angle was 25 degrees. The weather helm was felt on the tiller and the visual expectation of the leeward angle was around 5-8 degrees. The calculations based on the measurements through method $A$ for the same time interval gave an average value of AOA of keel of 3 degrees. This result seems wrong. The calculation was repeated with a simplified model, and the result was nearly the same. That points to the fact that either the hydrostatic, keel and rudder models, or both are wrong. Based on many tries, the error resides in the keel and rudder force estimation models, which seems not to be appropriate for this sailboat.

\subsection{Proposal for a New Sensor}

The weakness of this approach lies in the estimation of the AOA of the water hitting the rudder and the keel. The AOA, however, can vary with the depth along the keel because of the interference with the hull. The rudder is also subject to the interference from the keel preceding it. Such interference was modeled in (Larsson, Eliasson and Orych, 2014). The comparison of the present model and the model from (Larsson, Eliasson, \& Orych, 2014) solving by method A gives very similar results.

A cheap sensor for measuring the AOA on the keel would measure the leeway angle, and its design would be very similar to those used for the wind direction. A more sophisticated (and expensive) version is the directional ultrasound sensor which gives the water flow vector.

Following a series of measurements with the leeway angle sensor, it would be possible to create an ad-hoc expression that evaluates the sail forces. To validate the models, the measurements have to involve also an appropriate direct force measurement on the sail rig and sheet attachments, using strain gauges.

\subsection{Space for Improvement}

The inputs necessary to estimate the sail forces and torques in calm seas are:

1. roll angle

2. leeway angle

3. speed through the water

4. rudder angle

They can all be obtained from relatively cheap sensors.

A redundancy input would be the wind speed and wind angle that can be taken from anemometers found on many sailboats. This redundancy input can be used through an algorithm to estimate whether the sudden changes are due to wind, waves or crew movements.

Using the calculated values $F_{1,2}$, and guessed values for $d_{1,3}$ in the torque balance equation (35) will return a residual, which can be attributed to crew righting moment. 
Taking the measure of pitch and water pressure distribution on stations along the boat length allows to derive the hydrostatic moment and forces related to pitch by integration. This approach would allow to eliminate the holonomic constraint and to evaluate also the propulsive force deriving from surfing the waves ${ }^{2}$.

\section{REFERENCES}

AirFoil Tools web page, 2018. Available at: http://airfoiltools.com

Featherstone, R., 2008. Rigid Body Dynamics Algorithms. Available at: http://dx.doi.org/10.1007/978-1-4899-7560-7.
Fitzpatrick, R., 2018. Fluid mechanics teaching. Available at: http://farside.ph.utexas. edu/teaching/336L/Fluidhtml/node151.html

Fossati, F., 2009. Aero-Hydrodynamics and the performance of sailing yacht. Bloomsbury.

Larsson, L., Eliasson, R., and Orych, M., 2014. Principles of yacht design. McGraw-Hill Education - International Marine.

Mahne Kalin, A., 2014. Uporaba Xsens MTI/g senzorja pri meritvi dinamičnih karakteristik šolske jadrnice Solarije. Portorož, Slovenia: University of Ljubljana, Faculty of martime studies and transport.

Newman, J.N., 1977. Marine Hydrodynamics. MIT Press.

2. When buoyancy force is not acting vertically up. 